\title{
High-Speed Active Release End-Effector Motions for Precise Positioning of Adhered Micro-Objects
}

\author{
Eunhye Kim¹, Masaru Kojima1, Kazuto Kamiyama1, Mitsuhiro Horade1, \\ Yasushi Mae1, Tatsuo Arai'2,3 \\ ${ }^{1}$ Department of Systems Innovation, Graduate School of Engineering Science, Osaka University, Osaka, Japan \\ ${ }^{2}$ Beijing Advanced Innovation Center for Intelligent Robot and Systems, Beijing Institute of Technology, Beijing, China \\ ${ }^{3}$ The University of Electro-Communications, Tokyo, Japan \\ Email: kim@arai-lab.sys.es.osaka-u.ac.jp, kojima@arai-lab.sys.es.osaka-u.ac.jp
}

How to cite this paper: Kim, E., Kojima, M., Kamiyama, K., Horade, M., Mae, Y. and Arai, T. (2018) High-Speed Active Release End-Effector Motions for Precise Positioning of Adhered Micro-Objects. World Journal of Engineering and Technology, 6, 81-103.

https://doi.org/10.4236/wjet.2018.61005

Received: December 12, 2017

Accepted: January 29, 2018

Published: February 1, 2018

Copyright $\odot 2018$ by authors and Scientific Research Publishing Inc. This work is licensed under the Creative Commons Attribution International License (CC BY 4.0).

http://creativecommons.org/licenses/by/4.0/

\begin{abstract}
This paper presents a release method for micro-objects. To improve position accuracy after release, we propose 3D high-speed end-effector motions. The classical release task focuses on the detachment of a micro-object from an end-effector. The technique utilizes merely the vibration of the end-effector regardless of the pattern of movement. To release different sizes of microobjects and place them precisely at the desired locations in both air and liquid media, in this paper, we propose high-speed motions by analyzing the adhesion force and movement of micro-objects after separation. To generate high end-effector acceleration, many researchers have applied simple vibration by using an additional actuator. However, in our research, 3D high-speed motion with apt amplitude is accomplished by using only a compact parallel mechanism. To verify the advantages of the proposed motion, we compare five motions, 1D motions (in X-, Y-, and Z-directions) and circular motions (clockwise and counterclockwise directions), by changing the frequency and amplitude of the end-effector. Experiments are conducted with different sizes of microbeads and NIH3T3 cells. From these experiments, we conclude that a counterclockwise circular motion can release the objects precisely in air, while $1 \mathrm{D}$ motion in the $\mathrm{Y}$ direction and two circular motions can detach the objects at the desired positions after release in a liquid environment.
\end{abstract}

\section{Keywords}

Adhesion Force, Vibration Generation, Releasing Strategy, Micro-Manipulation 


\section{Introduction}

Manipulation of micro-scale objects is important for diverse applications in industrial, biological, and biomedical research such as in the assembly of biological cells for tissue engineering, development of sensing devices, and micro-surgical systems. In micromanipulation, the adhesion force between an end-effector and a micro-object is significantly different from macro manipulation, which is influenced by the gravitational force. The adhesion force includes van der Waals, electrostatic, capillary, and surface tension forces. These forces make it easy to pick up and transport an object, whereas the release of a micro-object is more difficult.

Diverse methods have been studied in order to release and place micro-scale objects precisely. The release methods can be classified into two strategies: passive release and active release [1]. Passive release techniques control the adhesion forces between the probe-object and object-substrate to detach the micro-object from the end-effector. For example, Saito utilized a glass needle and substrate coated with gold for grasping and releasing a $2 \mu \mathrm{m}$ polystyrene sphere to deal with adhesional and rolling-resistance factors [2]. Fuchiwaki et al. attached a 20 $\mu \mathrm{m}$ glass sphere to a glass needle with a ultraviolet-cured adhesive and detached the adhered object by irradiating the UV [3]. Hériban et al. selected a gel film (Gel-Pak) as substrate for accurate grasping and releasing of microparts [4]. Obviously, passive release is time-consuming and has poor repeatability. In addition, the method would not be suitable for controlling biological cells which depend mainly on surface properties. To overcome these disadvantages of the method, Horade et al. proposed an optimum end-effector having an uneven surface in order to release $20 \mu \mathrm{m}$ mouse fibroblast cells without adhesion [5]. The success rate of release was $85 \%$ with a position accuracy of a few microns. However, it was difficult to control the final position in a short time due to the water flow caused by the end-effector's motion, thus, slow operation for releasing was required.

On the other hand, active release methods apply external forces to detach micro-objects from an end-effector without making contact with the substrate. These methods reduce the adhesion force by using pressure changes (vacuum tools) [6], voltage control [7], and dynamic effects (vibration) [8]-[13]. Among existing methods for active release, dynamic effects, i.e., the release of microobjects from an end-effector by vibrating the end-effector to generate inertial force, has drawn much attention recently owing to its high success rate. However, dynamic release still has problems, including difficulty in object control after detachment. To solve these problems, many researchers have proposed various solutions. Fang et al. calculated and simulated the landing radius of the released object according to the vibration amplitude and the frequency of the end-effector [14]. B. K. Chen et al. utilized a plunging mechanism to generate sufficient momentum. They detached $7.5-10.9 \mu \mathrm{m}$ borosilicate glass spheres with an accuracy of $0.7 \pm 0.46 \mu \mathrm{m}$ [15]. Watanabe et al. proposed a squeezing effect for preci- 
sion micromanipulation by vibrating a finger and squeezing a film [16]. However, it is necessary to control precisely the displacement of the end-effector and additional equipment of the measurement system for checking the distance between the micro-object and the end-effector. T. Chen et al. suggested an active release strategy based on adhesion control with compound vibration [9]. The success rate of release was $100 \%$ with an accuracy of $4 \pm 0.5 \mu \mathrm{m}$. However, they applied a special mechanism with two arms controlled by an electrostatic actuator and a piezoelectric (PZT) actuator to apply these methods. Rong et al. separated $35-100 \mu \mathrm{m}$ microbeads from an end-effector with $4.5 \pm 0.5 \mu \mathrm{m}$ position accuracy by using vibration release with a vacuum tool [6].

In this paper, we propose an active release technique using high-speed endeffector motions controlled by a parallel mechanism. The purpose of this research is the release of micro-objects at the desired positions with high placement accuracy by comparing several end-effector motions. Previous dynamic release studies utilized additional mechanisms to generate sufficient acceleration. However, we present a parallel-link mechanism with three degrees of freedom actuated by three PZT actuators. Using this parallel mechanism, 3D end-effector motion at high speed is feasible. Previous experiments using this mechanism from [8] focused on generating the necessary acceleration in order to release various sizes of micro-objects by changing frequencies with a fixed amplitude (1 $\mu \mathrm{m})$ of the end-effector. However, this research places an emphasis on motions of the end-effector that can control placing positions after release. To be specific, we analyze the force during and after release of micro-objects in air and liquid media and apply it to motions that can achieve precise positioning with small positional change after release. We propose a circular motion in the $\mathrm{XZ}$ plane by combining the oscillation of the end-effector in the X-and Z-directions in an atmospheric environment as well as circular motion and $1 \mathrm{D}$ motion in the $\mathrm{Y}$-direction in a liquid environment. To verify the advantages of using the proposed motions, three $1 \mathrm{D}$ motions (X, Y, and $\mathrm{Z}$ ) and two circular motions (clockwise and counterclockwise) are tested together in each condition (air and liquid). An early version of the research presents preliminary experimental results [17]. The current study is notably improved and can be a progress paper for releasing and accurate placing of biological objects by high-speed end-effector motions. The main enhancements of this paper are following: 1) analysis of high-speed motions to release object at the desired position; 2) additional experimental data using various sizes of objects and biological cells with different environments; and 3) statistical analysis of experimental results and further discussion.

The following section describes the release strategy for precise placement. In the third section, an experimental system for releasing micro-objects is explained. The last section presents experimental results by comparing several high-speed motions in different environments. The results show that the proposed motions of the end-effector can release micro-objects at the desired posi- 
tions with high placement accuracy.

\section{Release Strategy for Precise Positioning}

\subsection{Adhesion Effect on Micromanipulation}

In micromanipulation, the most difficult problem is probably caused by the adhesion effect. To overcome the adhesion phenomenon, the van der Waals, capillary, and electrostatic forces should be taken into account [18] [19] [20]. The dynamic release method, which primarily takes advantage of inertial effects, is applied to reduce the adhesion force [21]. One limitation of dynamic release is its difficulty in positioning objects after release. The release task is divided into two stages: when the object is in contact with the end-effector and after the object has detached from the end-effector. During the first stage, to separate an object that is in contact, the minimum amount of force is required, which is called the pull-off force.

$$
F_{\text {ext }}=m_{p} a>\frac{3}{2} \pi R_{b} \gamma_{b p}
$$

$F_{\text {ext }}$ in (1) from the end-effector should be made larger than the pull-off force by applying the Johnson Kendall Roberts (JKR) contact model for overbalancing the adhesion force between an end-effector and a micro-object [14]. $R_{b}$ represents the radius of the micro-object, and the work of adhesion between the object and the end-effector $\left(F_{\text {ext }}\right)$ can be calculated by the surface forces of the object and the end-effector. The schematic explanation is shown in Figure 1.

The computation of the external force $\left(F_{\text {ext }}\right)$ is dependent on the movement of the end-effector. When the acceleration of the end-effector (a) is high enough, the object can be separated from the end-effector. However, to release the object at a suitable position with small landing area, the control of the release motion is necessary because a large amount of external force cannot guarantee position accuracy after separation [14]. To estimate the pull-off force, we assume several parameters based on the experimental conditions and [22]. In our system, a glass needle and polystyrene spheres with a diameter of $55 \mu \mathrm{m}$ are utilized for an

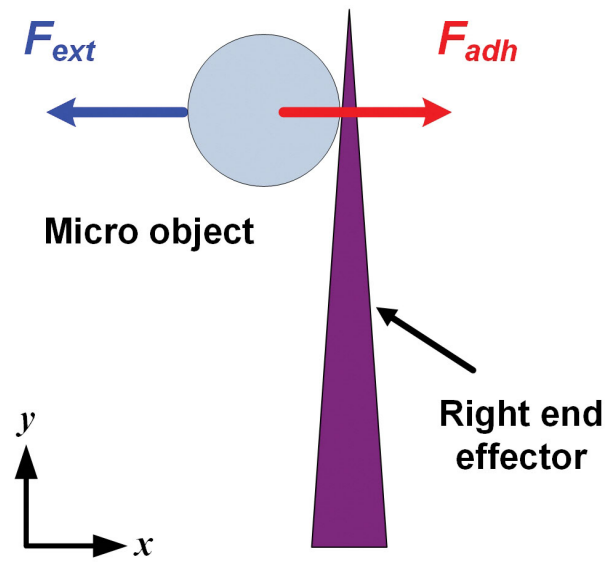

Figure 1. Adhesion force between an object and an end-effector. 
end-effector and a target object, respectively. Based on the assumed parameters, the pull-off forces are calculated to be $2.03 \times 10^{-5} \mathrm{~N}$ in air and $5.37 \times 10^{-6} \mathrm{~N}$ in water.

\subsection{High-Speed Motions for Release}

To verify the successful release and precise positioning of an object when using the proposed motions, five motions, including circular motions (clockwise and counterclockwise) and 1D motions (X, Y, and Z) were compared. Figure 2 shows five high-speed motions of the right end-effector. The red arrows in the figure represent the direction of each motion. To illustrate the shape of motions clearly, we draw each motion in different views, for example, Figure 2(a) and Figure 2(b) in XY plane, Figure 2(c) in YZ plane, and Figure 2(d) and Figure 2 (e) in 3D space. Figures 2(a)-(c) describe three 1D motions in the X-, Y-, and Z-directions. On the other hand, Figure 2(d) \& Figure 2(e) displays circular motions in the clockwise and the counterclockwise directions.

\section{Circular Motion for Precise Release}

One of the important considerations in releasing an adhered object on the planned position is to reduce the velocity of the object before it reaches the substrate. In a micro-scale environment, although the inertial forces from the endeffector's motion are very small, the accelerations of micro-objects are usually very high. Subsequently, the velocity of the object is able to increase in a very short time and the trajectory of the object could be difficult to control. Finally, the object can jump rapidly out of the visible area. The attachment position between the end-effector and the micro-object are also vital factors for the trajectory of the object after separation. The high-speed camera provides the position of the target object and end-effectors; however, 3D position information, including Z-position, is generally difficult to acquire in a micro-environment. For
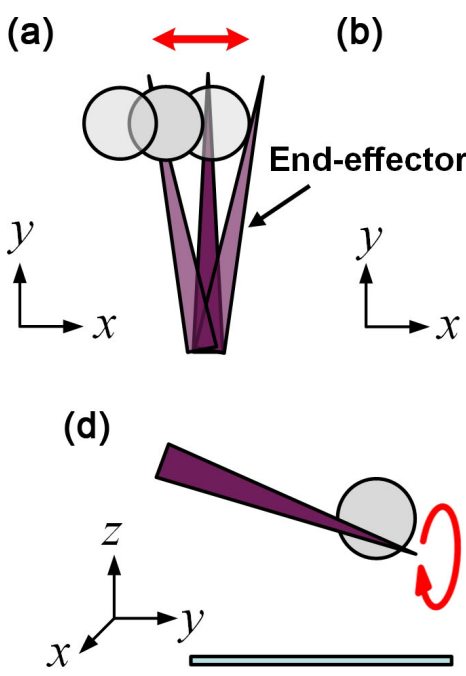

(b)

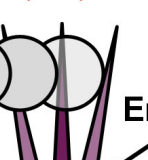

End-effector
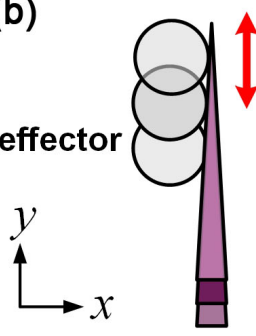

(c)

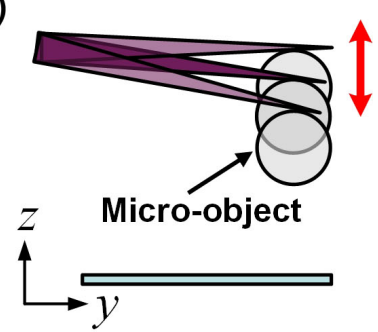

(e)

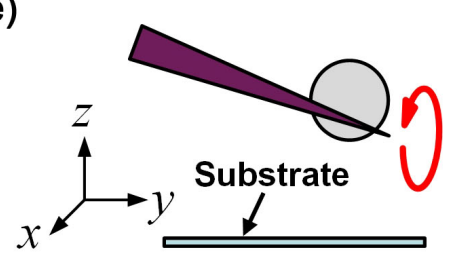

Figure 2. High-speed motions of the end-effector (a) 1D motion (X-axis) (b) 1D motion (Y-axis) (c) 1D motion (Z-axis) (d) circular motion (CW) (e) circular motion (CCW). 
(a)

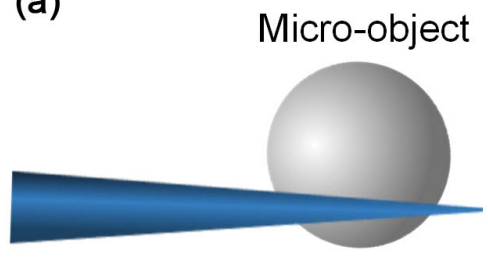

End-effector (b)

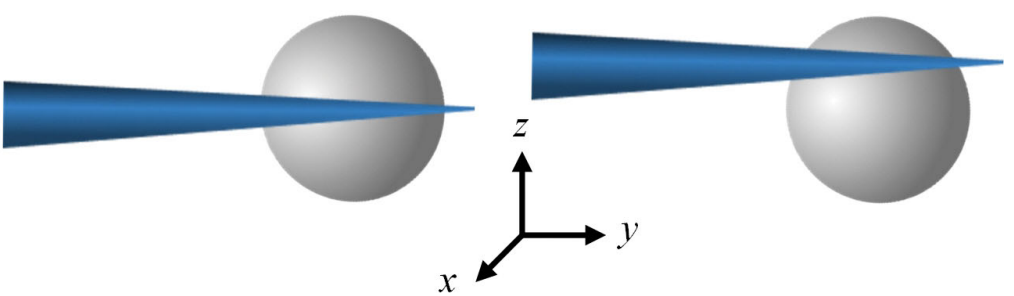

Figure 3. Adhered position of an object before separation (a) adhered to the lower part (b) adhered to the center part (c) adhered to the upper part.

this reason, objects can be adhered to an end-effector in several patterns as shown in Figure 3. Owing to these difficulties, the prediction of an object's landing trajectory is excessively complex. Therefore, a special motion that can release objects into a small landing area regardless of the contact position is needed. In this paper, circular motion is proposed for precise release.

To compare the velocity of the released object, we calculate the acceleration of the end-effector according to the motions. 1D motions that can be composed of the sinusoidal curve generate an acceleration which is directly proportional to the displacement in opposing direction. On the other hand, circular motions create a constant speed by changing the direction, which means that the object is accelerating (centripetal acceleration). However, since the initial speed of the motion is zero, we should consider the tangential acceleration and centripetal acceleration together until the desired speed is reached. In this section, the main assumption in order to compare two motions is that the maximum velocity and increased time to maximum velocity of two motions are same. In addition, the amplitude $(A)$ and the angular velocity $\left(\omega_{n}\right)$ of two motions are equal. Accelerations of the 1D motion and the circular motion can be calculated by Equations ((2) and (3)), respectively. $A$ indicates the amplitude and $\omega_{n}$ is the angular velocity of the end-effector's motion. According to Equation (2), when the displacement is maximum, the acceleration is maximum. Moreover, the maximum acceleration of the $1 \mathrm{D}$ motion is same as the centripetal acceleration of the circular motion calculated by Equation (3) if $t>t_{1}$. The acceleration of circular motions can be divided by the time $\left(t_{1}\right)$ when the end-effector reaches the maximum velocity. The angular velocity is varied from 0 to $\omega_{n}$ until $t_{1}$, so it relies on the time ( $\mathrm{t})$. After $t_{1}$, the angular velocity is fixed as $\omega^{2}$ because the end-effector makes a uniform circular motion. To estimate the acceleration of two different motions, we assume several parameters. For example, $f_{n}$ is assumed to be $100 \mathrm{~Hz}$ and $A$ is assumed to be $3 \mu \mathrm{m}$. Thus, the maximum velocity and the time to reach the maximum velocity of the $1 \mathrm{D}$ motion are calculated to be $1.9 \times 10^{-3} \mathrm{~m} / \mathrm{s}$ and $5 \mathrm{~ms}$, respectively. Applying these values, we can compute the acceleration of the circular motion until the time $t_{1}$. At that time, the endeffector accelerates to the desired speed $\left(1.9 \times 10^{-3} \mathrm{~m} / \mathrm{s}\right)$ and $\theta$ is changed from $0^{\circ}$ to $180^{\circ}$ in the time interval of $5 \mathrm{~ms}\left(t_{1}\right)$. Thus, the tangential acceleration and centripetal acceleration of the circular motion when $t_{1}$ are calculated to be 0.38 
$\mathrm{m} / \mathrm{s}^{2}$ and $1.18 \mathrm{~m} / \mathrm{s}^{2}$, respectively. After $t_{1}$, the uniform circular motion can be formed, as a result, the centrifugal acceleration of the circular motion and the maximum acceleration of the $1 \mathrm{D}$ motion are calculated to be $1.18 \mathrm{~m} / \mathrm{s}^{2}$. The external forces $\left(F_{\text {ext }}\right.$ ) also can be computed by using Equation (1). $m_{p}$ is assumed to be $2.4 \times 10^{-5} \mathrm{~kg}$. Therefore, the external force of the circular motion is $2.98 \times$ $10^{-5} \mathrm{~N}$ when ${ }^{t_{1}}$ and $2.84 \times 10^{-5} \mathrm{~N}$ after $t_{1}$, whereas, the force of the $1 \mathrm{D}$ motion is constantly changed from $0 \mathrm{~N}$ to $2.84 \times 10^{-5} \mathrm{~N}$. As a result, the maximum force of the $1 \mathrm{D}$ motion is identical with the force of the uniform circular motion.

$$
a_{1}=\omega_{1}^{2} x
$$

where, $x=A \sin \left(\omega_{1} t\right), \omega_{1}=2 \pi f_{1}$

$$
a_{2}= \begin{cases}\sqrt{(\ddot{\theta} A)^{2}+\left(\dot{\theta}^{2} A\right)^{2}}, & t \leq t_{1} \\ \omega_{2}^{2} A, & t>t_{1}\end{cases}
$$

where, $\omega_{2}=2 \pi f_{2}$

From estimated values, we can assume trajectories of the released object by two types of motions. Not only to detach the object from the end-effector, but also to reduce the velocity of the object after release, the minimum amount of forces should be applied. In case of the 1D motion, when the end-effector reaches the maximum displacement, the maximum force is applied and then the object will be released. On the other hand, the circular motion produces the same amount of forces before $t_{1}$, thus the object will be released at that time. Obviously, the magnitude of the exerted force of the two motions when the object is released is same as mentioned above. However, the velocity of the object after release can be guessed by considering the direction of applied forces. The force of the $1 \mathrm{D}$ motion is exerted on the adhered object in the same direction as the end-effector's motion, so the object will be released in the same direction of the motion. In contrast, an object adhered to the end-effector that moves in a circular path experiences the centrifugal force directed toward the center of the circular motion and the tangential force. If the object is released, the centrifugal force will be vanished. Subsequently, the object will travel in the tangential direction with a constant speed. Thus, the force for release of the object is slightly reduced, which makes the objects travel shorter distance than the 1D motion.

In addition, the circular motion can release object into the small region in spite of the different contact position. For instance, in case of the 1D motion in the X-direction, when the object adhered to the center part like Figure 3(b), the object will be moved in a straight line, horizontal to the $\mathrm{X}$-axis. However, when the object attached to the lower or the upper part like Figure 3(a) and Figure $3(c)$, the moving direction of the object is changed according to the adhered angle between end-effector and the center of the object. Thus, it is difficult to estimate the final position of the object. On the other hand, in case of the circular motion, the released object will be moved in a straight line tangent to the circle at the point where the object is separated. Thus, the moving direction of the object is not significantly affected in the contact position (Figure 3). Moreover, 
both types of motions can be dependent on the frequency and the amplitude of the end-effector. As the frequency and the amplitude increase, the external forces will be increased, and subsequently, the object released from the strong external force will travel longer distance.

Based on the applied force and the direction of the motion, we can estimate the movement of the object after release. 1D motion in the X-direction makes an object move in a straight line in the X-direction, therefore, the object travels with further displacement than the proposed motions and may even be located outside of the workspace. The object released by the 1D motion in Y-direction could be not only dropped into the substrate but also moved in the Y-direction with high velocity. Finally, the object also travels a long distance. The object manipulated by $1 \mathrm{D}$ motion in $\mathrm{Z}$-direction could be released in a straight line in the $\mathrm{Z}$-direction and be finally located at the nearest position. However, the object can reach the substrate having high velocity due to the high acceleration and then the object can sometime bump into the substrate rapidly. In contrast, circular motion creates an arc trajectory for an object. As an object moves in circular motion, it constantly changes its direction. After the object is released, it moves in a straight line at constant speed, tangent to the circle at the point where the object is located. Subsequently, the object moves in a parabolic path after release, causing the object to reach the substrate at a lower velocity than the former motions.

In this paper, we conduct the release task in air and liquid media together. $F_{d}$ in Equation (4) indicates the drag force of the fluid on a sphere. $\mu$ represents the dynamic viscosity of the medium (air: $1.85 \times 10^{-5}$, water: $1 \times 10^{-3} \mathrm{~Pa} \cdot \mathrm{s}$ ) and $V$ is the relative velocity of the fluid with respect to the object.

$$
F_{d}=-6 \pi \mu R_{b} V
$$

The drag force is able to reduce the velocity of the object to be applied in the opposite direction. In water, the dynamic viscosity has a stronger influence on the drag force of the fluid than in air. As the result, an object in water will be located at a closer position than in air in spite of the same external forces thanks to the high drag forces [22]. Nevertheless, motion in only one direction cannot ensure precise positioning after release in an air environment for the reasons mentioned. By analyzing the movement of objects after release, we conclude that the circular motion is better solution for precise releasing of micro-object than the 1D motion. This motion can release objects into the small landing areas in both media by using the centrifugal force, in addition, the motion can release object precisely regardless of the contact position thanks to the direction of the force.

\section{Experimental Setup}

\subsection{Overall System for Micro-Manipulation}

In this system, a two-fingered micro-hand was utilized for grasping, transporting, and releasing objects. The micro-hand is displayed in Figure 4. Manipulation of micro-objects was accomplished by two operating systems: a Linux PC 


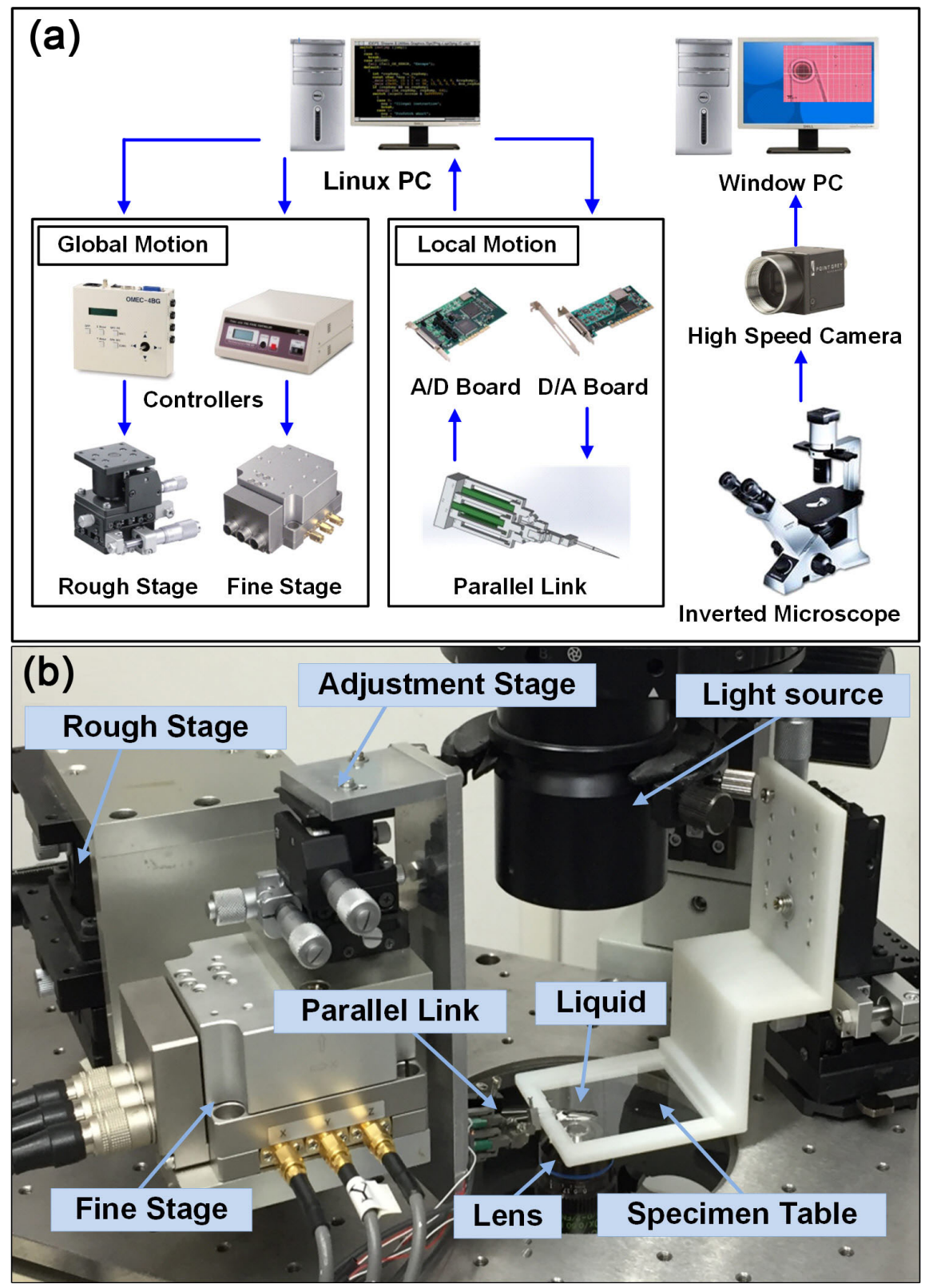

Figure 4. Two-fingered micro-hand (a) configuration of the micro-manipulation system (b) overall micro-hand system.

(Dell, XPS600, Pentium 4 at $3.80 \mathrm{GHz}$ ) and a Windows PC (Intel Core i7 CPU at $2.93 \mathrm{GHz}$ with $4 \mathrm{~GB}$ of RAM). The Linux PC was in charge of manipulating the micro-hand. The motion of the micro-hand was composed by two parts: a global motion and a local motion. The global motion handled the movement of both end-effectors for transporting target objects and positioning the micro-hand. Two motorized stages, a rough stage (Sigma-Koki, TSD-805S) and a fine stage (Sigma-Koki, SFS-H60XYZ), made the micro-hand move in a large workspace with precision positioning. The rough stage actuated by three DC motors was controlled by a stage controller (Sigma-Koki, OMEC-4BG). On the other hand, the fine stage driven by piezo actuators was managed by a controller (Sigma- 
Koki, Fine-503).

Local motion manipulated the right end-effector controlled by a compact parallel link for grasping and releasing variously sized objects. The displacements of three PZT actuators (NEC TOKIN, AE0203D16) determined the 3D position of the right end-effector through a D/A board (Contec DA16-16(LPCI)L) and an amplifier (MATSUSADA, HJPZ-0.15Px3). For measuring the displacement of the PZT actuators, strain gages were implemented. In addition, the measured outcomes were transferred to the Linux PC through an amplifier (Kyowa MCD$16 \mathrm{~A}$ ) and $\mathrm{A} / \mathrm{D}$ converter (Contec AD16-16(PCI)EV). One adjustment stage, operated manually for adjusting the right end-effector with respect to the local motion, moved $6 \mathrm{~mm}$ in a 3D direction with a resolution of $3 \mu \mathrm{m}$. To realize a large distance between the two end-effectors for grasping tasks, the manual stage was necessary. The two end-effectors of the micro-hand and the target objects were observed under an IX81 motorized inverted optical microscope using an objective lens. The Windows PC displayed the images of the two end-effectors and objects captured by the high-speed camera (Photron FASTCAM MC2). Two fine-tipped glass needles, having a 23-mm length, 1-mm diameter, and tips with less than 1- $\mu \mathrm{m}$ curvature, were mounted in the micro-hand at the end of the parallel link. The structure of two-fingered micro-hand is shown in Figure 5.

\subsection{Structure of a Parallel Mechanism}

In order to detach diverse sizes of objects from the end-effector at the desired locations, the 3D high-speed motions without uncontrollable vibration were applied by using a parallel mechanism. The parallel manipulator was stiffer, faster, and more accurate than the serial mechanism. Therefore, we utilized the parallel mechanism to achieve 3D high-speed motions with precise positioning. The concise parallel link was a modified version of a previous structure [23], which used 3 PRR (prismatic-revolute-revolute) parallel mechanisms. Three PZT actuators were employed as prismatic joints that could be extended up to $10.7 \mu \mathrm{m}$, which yielded three degrees of freedom: two rotational in the X- and Z-directions and one translational in the Y-direction. A CAD image and the structure of the proposed model are described in Figure 6(a).

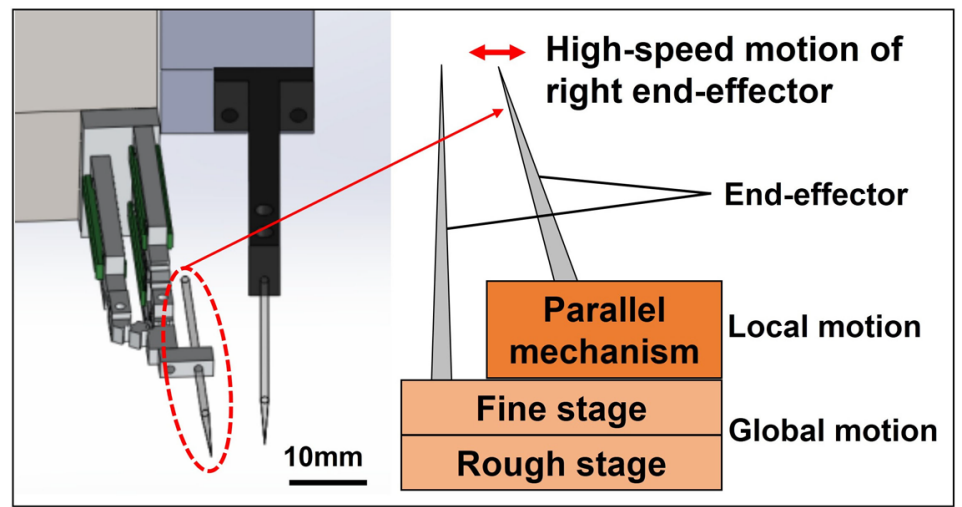

Figure 5. Structure of two-fingered micro-hand. 
(a)

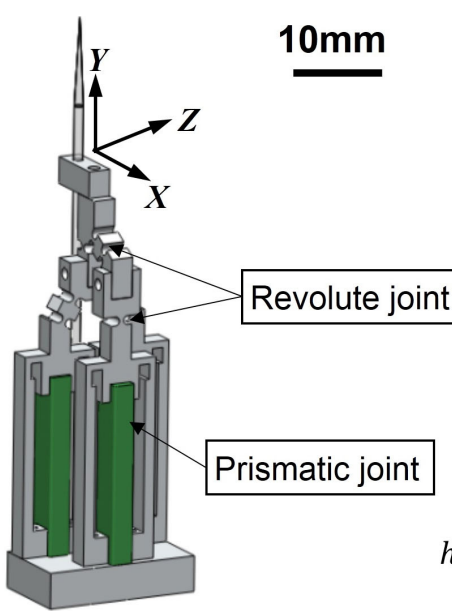

(b)

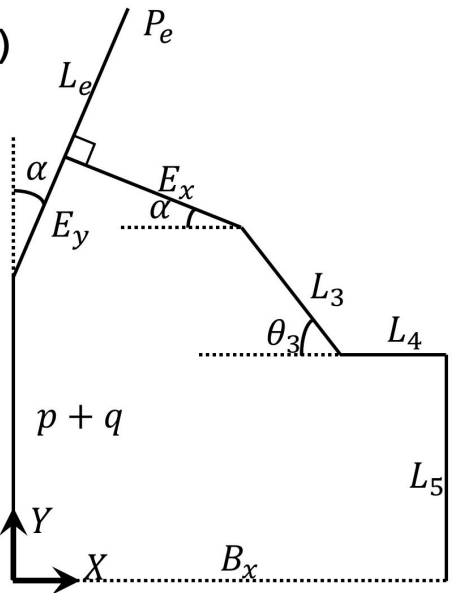

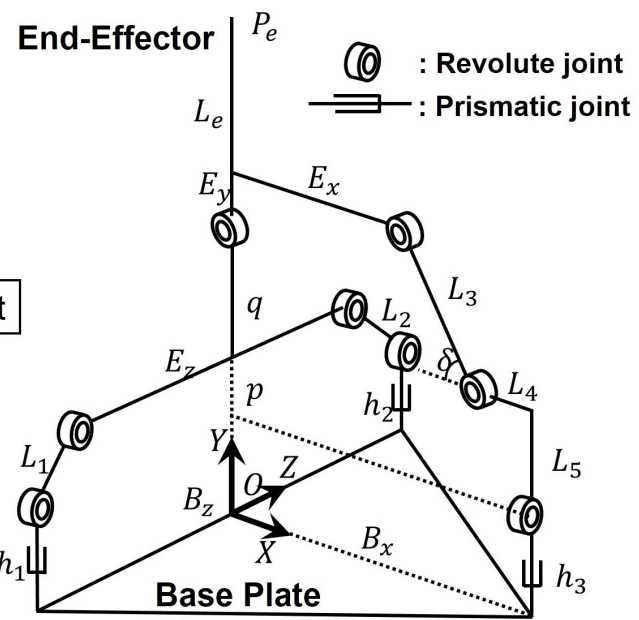

(c)

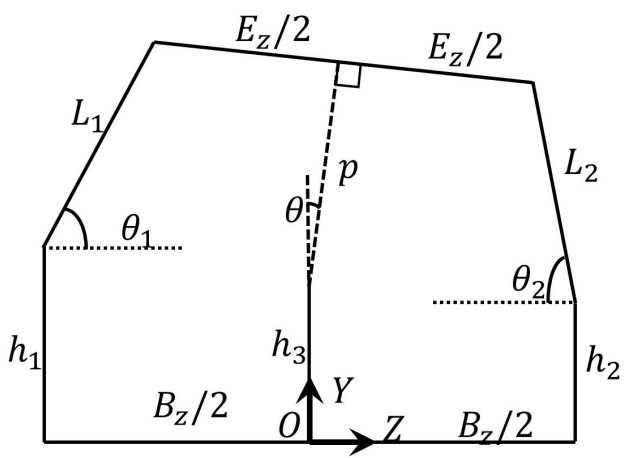

Figure 6. Compact parallel link (a) structure of the parallel mechanism (b) the mechanism in the plane defined by $p$ and the axis of the revolute joint at $h_{3}(\mathrm{c})$ alignment of the linakge in the $\mathrm{YZ}$ plane.

To analyze the workspace of the parallel mechanism, inverse kinematics and structural analysis are utilized. In inverse kinematics of the mechanism, the length of piezo actuators $\left(h_{1}, h_{2}\right.$, and $\left.h_{3}\right)$ can be solved from a position of the end-effector $P_{e}\left(P_{e x}, P_{e y}\right.$, and $\left.P_{e z}\right)$. The proposed mechanism in the plane defined by $p$ and the axis of the revolute joint located a distance $h_{3}$ is displayed in Figure 6(b). The linkage alignment of the model on the $\mathrm{YZ}$ plane is shown in Figure 6(c). Applying the given values in Figure 6, the position of the end-effector can be computed.

$$
\begin{gathered}
P_{e x}=\left(E_{y}+L_{e}\right) \sin \alpha \\
P_{e y}=h_{3}+\left\{p+q+\left(E_{y}+L_{e}\right) \cos \alpha\right\} \cos \theta \\
P_{e z}=\left\{p+q+\left(E_{y}+L_{e}\right) \cos \alpha\right\} \sin \theta
\end{gathered}
$$

In this equation, we set $L_{e}$ to an extension of $E_{z}$. Based on Figure 6(b), Equation (8) and Equation (9) are expressed as follows:

$$
E_{y} \sin \alpha+E_{x} \cos \alpha+L_{3} \cos \theta_{3}+L_{4}=B_{x}
$$




$$
p+q+E_{y} \cos \alpha=E_{x} \sin \alpha+L_{3} \sin \theta_{3}+L_{5}
$$

With all of the above results, Equations ((5) and (6)), it is possible to find the length of the piezo actuator $\left(h_{3}\right)$. The angle $\alpha$ is calculated by Equation (5), and the angle $\theta_{3}$ is obtained by substituting $\alpha$ into Equation (8). $p$ and $\theta$ are calculated by Equations ((9) and (7)), respectively. Finally, the value of $h_{3}$ can be solved by substituting the above parameters into Equation (6).

To solve $h_{1}$ and $h_{2}$, we concentrate on two polygons, divided by the $p$ in Figure 6(c). Equations ((10) and (11)) indicate the equation of each axial direction in the left polygon. On the other hand, Equations ((12) and (13)) are calculated by the right polygon.

$$
\begin{gathered}
L_{1} \cos \theta_{1}+E_{z} \cos \theta / 2=B_{z} / 2+p \sin \theta \\
h_{1}+L_{1} \sin \theta_{1}=h_{3}+p \cos \theta+E_{z} \sin \theta / 2 \\
L_{2} \cos \theta_{2}+E_{z} \cos \theta+p \cos \theta=B_{z} / 2 \\
h_{2}+L_{2} \sin \theta_{2}+E_{z} \sin \theta=h_{3}+p \cos \theta
\end{gathered}
$$

To find $h_{1}, h_{2}$ and $h_{3}$, the angles $\theta_{1}, \theta_{2}$, and $\theta_{3}$ are computed by using Equations ((10), (12), and (8)), respectively.

$$
\begin{gathered}
\theta_{1}=\cos ^{-1}\left(\left(B_{z} / 2+p \sin \theta-E_{z} \cos \theta / 2\right) / L_{1}\right) \\
\theta_{2}=\cos ^{-1}\left(\left(B_{z} / 2-p \sin \theta-E_{z} \cos \theta / 2\right) / L_{2}\right) \\
\theta_{3}=\cos ^{-1}\left(\left(B_{x}-L_{4}-E_{y} \sin \alpha-E_{x} \cos \alpha\right) / L_{3}\right)
\end{gathered}
$$

Finally, the value of $h_{1}, h_{2}$ and $h_{3}$ can be found in terms of $P_{e}$. With all of the calculated results, we find the piezo actuators values in the parallel link.

Based on the above calculations, we get the axial displacement of the workspace. To be specific, the displacement in X-direction is $128 \mu \mathrm{m}(-64 \sim 64 \mu \mathrm{m})$ and in $\mathrm{Y}$-direction is $17 \mu \mathrm{m}(0 \sim 17 \mu \mathrm{m})$. In the Z-direction, the end-effector can move $110 \mu \mathrm{m}(-55 \sim 55 \mu \mathrm{m})$.

\subsection{Controllable Motion Using a Parallel Link}

To control the movement of the right end-effector precisely, the relationship between the parallel link extension using the strain gages and the positional change of the end-effector using the camera was investigated. The $3 \mathrm{D}$ position of the right end-effector managed by the piezo-actuated parallel link was influenced by the length of the end-effector. Thus, at first, we set the length of the end-effector to $16 \mathrm{~mm}$ and subsequently performed calibration by obtaining a calibration matrix calculated by the linear relationship between the displacement of the PZT actuators and the positional change of the end-effector in three directions. Finally, we set the displacement of the end-effector to about $80 \mu \mathrm{m}$ in the $\mathrm{X}$ - and Z-directions and $10 \mu \mathrm{m}$ in the Y-direction. In our system, the displacement of the $\mathrm{Y}$-axis was smaller than in the other directions owing to the mechanism; the movement in the Y-direction was determined by the length of the PZT actuators because it was generated by moving the three piezo actuators, 


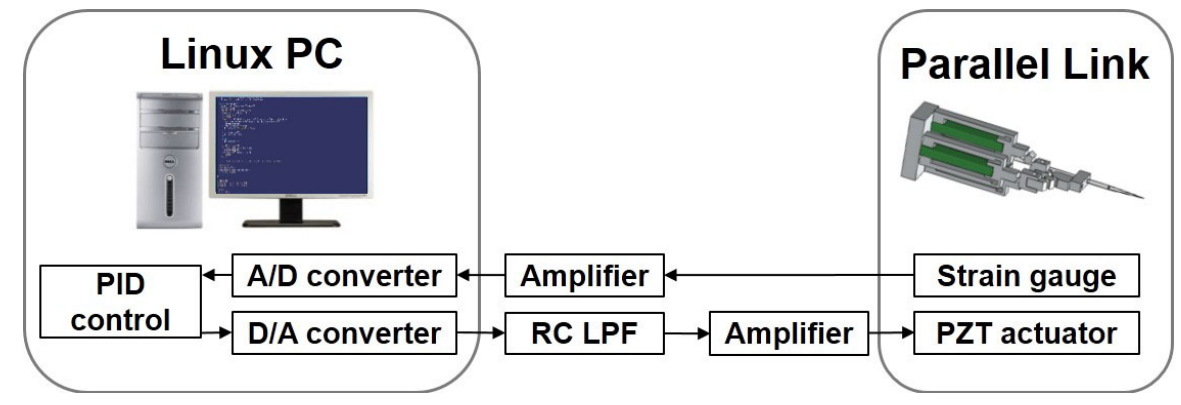

Figure 7. Control scheme for the parallel link.

which could stretch to $10.7 \mu \mathrm{m}$ simultaneously.

To generate enough force to separate the micro-objects, high-speed motions of the end-effector at high frequency (about $1 \mathrm{kHz}$ ) and amplitude were required. The control scheme for the parallel link is shown in Figure 7. The values of strain gages measuring the extension of the PZT actuators were transmitted to the Linux PC trough an amplifier and an A/D converter. Applying the received sensor values, the orientation and position of the end-effector were computed. To move the end-effector to the desired position, the proper voltage determined by the computed data was applied to each PZT actuator via a D/A converter and an amplifier. In addition, to reduce uncontrollable oscillations at high speeds, a PID controller for feedback control and an RC circuit as a Low-Pass Filter were added. As a result, we succeed in decreasing irregular movement at the highest frequency $(1 \mathrm{kHz})$ from over $5 \mu \mathrm{m}$ to less than $0.5 \mu \mathrm{m}$. In this system, the displacement of the end-effector was reduced during high-speed motion. At the maximum frequency $(1 \mathrm{kHz})$, the amplitude was decreased by 30 percent from the calibrated values.

Using this parallel link, we generated five types of high-speed motions Figure 2. $1 \mathrm{D}$ motions in the $\mathrm{X}$-, $\mathrm{Y}$-, and Z-directions consisted of step-functions with two steps. On the other hand, circular motions in the clockwise and the counterclockwise directions composed of the combination of a sine function in the $\mathrm{X}$-direction and a cosine function in the Z-direction with ten steps. Owing to the number of steps and the highest frequency of the parallel link for a step $(1 \mathrm{kHz})$, the maximum frequency for the five motions was $100 \mathrm{~Hz}$.

\subsection{Manipulation of Various Sizes of Objects}

This experiment aimed to release micro-objects at the desired positions using a right end-effector controlled by a parallel link mechanism. For this experiment, 10-, 25-, and 55- $\mu \mathrm{m}$ microbeads and NIH3T3 cells $(16 \pm 2 \mu \mathrm{m})$ were utilized as the target objects. According to the sizes of the objects, different sizes of lens were used. For the $55-\mu \mathrm{m}$ microbeads, a $10 \times$ objective lens was used, allowing a visible space of $512 \mu \mathrm{m} \times 512 \mu \mathrm{m}$. $25-\mu \mathrm{m}$ microbeads and biological cells were utilized with a $20 \times$ objective lens, allowing a visible space of $256 \mu \mathrm{m} \times 256 \mu \mathrm{m}$. On the other hand, for the $10-\mu \mathrm{m}$ microbeads, a $40 \times$ objective lens was applied, providing a visual space of $128 \mu \mathrm{m} \times 128 \mu \mathrm{m}$. 
A high-speed camera capable of capturing images at 2000 frames per second to visualize the high-speed motion was applied. Because the maximum frequency of a step in the motions studied was $1 \mathrm{kHz}$, in order to capture images that identify the direction and the speed of the objects, a high-speed camera that could capture images at over 1000 frames per second was necessary. The release experiments were performed at $20 \mu \mathrm{m}$ above the substrate to avoid being affected by the motions of the end-effector. Each motion was repeated 8 times, and the performance duration for each motion was $100 \mathrm{~ms}$. Room temperature was set to $24^{\circ} \mathrm{C}$. The experiment was performed in different environments; for example, with 55- $\mu \mathrm{m}$ microbeads in air, 10-, 25-, 55- $\mu \mathrm{m}$ microbeads in water, and $16-\mu \mathrm{m}$ NIH3T3 cells in phosphate-buffered saline (PBS).

Figure 8 shows the manipulation process for a $55-\mu \mathrm{m}$ microbead. Figure 8 (a) and Figure 8(b) show the grasping task, which included moving both end-effectors close enough to the target object and moving the right end-effector to grasp the object. Figure 8 (c) shows a release attempt without using any special method for moving the right end-effector. The object was then attached to the right end-effector. To release an object for precise positioning, the release height was a vital factor because it determined location accuracy after release [6] [9]. In this experiment, the release height was fixed to $20 \mu \mathrm{m}$ by changing the $\mathrm{Z}$-positions of both end-effectors (Figure $8(\mathrm{~d})$ ). The object sometimes bumped into the left end-effector after release. To prevent collisions between the object and the left end-effector during and after release, the right end-effector was moved manually by using the adjustment stage (Figure $8(\mathrm{e})$ ). Finally, the release task using highspeed motions were performed (Figure $8(\mathrm{f})$ ).

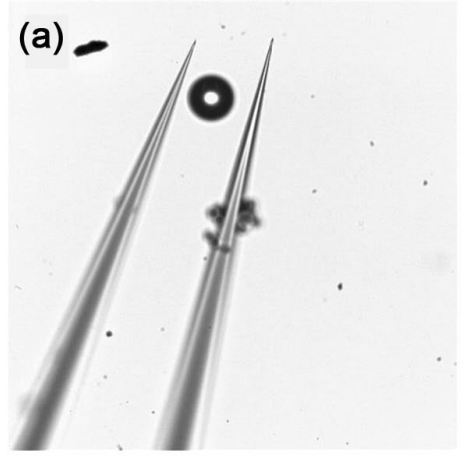

(d)

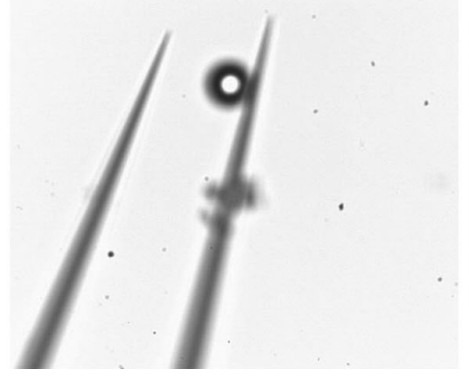

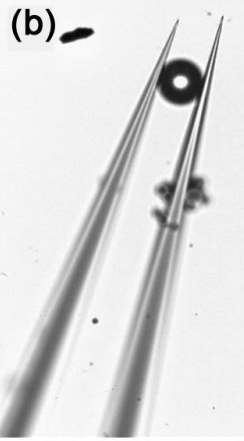

(e)

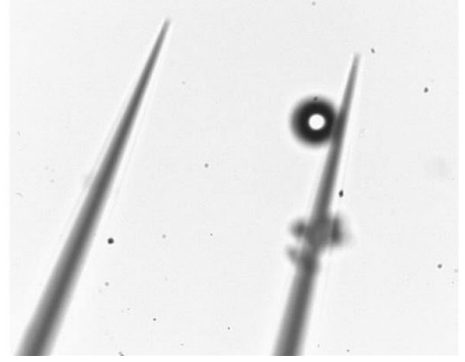

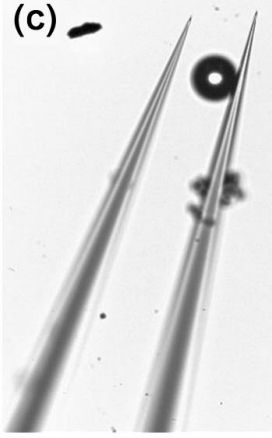

(f)

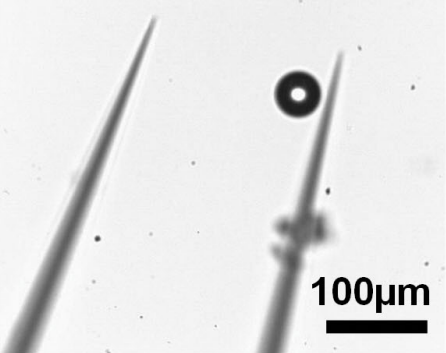

Figure 8. Manipulation process for a $55-\mu \mathrm{m}$ microbead. 


\section{Experimental Results and Discussion}

\subsection{Release Conditions for Different Objects}

According to the sizes of objects and the environment, the range of frequency and amplitude of the end-effector that could release the adhered object was different. To determine the possible frequency and amplitude of the end-effector for release in different environments and different object sizes, the maximum frequency was applied to adhered micro-objects by changing the amplitudes of the end-effector; then, lower frequencies were tested using the same values of amplitude. If the release of microbeads was not achieved or if the object moved outside of the visible area every time, these cases were excluded from the experiment. The ranges of amplitudes and frequencies for the end-effector using circular motions according for different objects in different environments under the proposed conditions are shown in Table 1.

Specifically, at first, experiments using $55-\mu \mathrm{m}$ microbeads in air were performed at the maximum frequency $(100 \mathrm{~Hz})$. As a result, when the amplitude of the end-effector was smaller than $4 \mu \mathrm{m}$, objects did not detach from the end-effector. On the other hand, when the amplitude was larger than $9 \mu \mathrm{m}$, objects flew outside of the visible space. Thus, we decided that the useful range of the amplitude of the end-effector was from $4 \mu \mathrm{m}$ to $9 \mu \mathrm{m}$ except for $1 \mathrm{D}$ motion in the Y-direction. Thereafter, we applied this range of amplitudes at different frequencies $(50,25,20$, and $10 \mathrm{~Hz})$. If the objects were released successfully at the same conditions, the frequency was selected. Finally, we determined that the frequencies of the motions were $50 \mathrm{~Hz}$ and $100 \mathrm{~Hz}$. However, in the case of 1D motion in the Y-direction, only two amplitudes $(1.2 \mu \mathrm{m}$ and $2.4 \mu \mathrm{m})$ were applied because the maximum movement of the end-effector in the $\mathrm{Y}$ direction was $2.4 \mu \mathrm{m}$ at $100 \mathrm{~Hz}$.

In order to compare the release conditions between air and liquid environments, the same range of amplitudes $(4 \sim 9 \mu \mathrm{m})$ and frequencies $(50 \mathrm{~Hz}$ and 100 $\mathrm{Hz}$ ) in air were applied in the liquid environment. In the liquid environment, the objects were always located in the visible area (in contrast with the case in air); on the other hand, the adhered objects could detach from the end-effector when the amplitude or frequency were too low. For instance, $55-\mu \mathrm{m}$ microbeads in water detached from the end-effector under the same conditions (amplitudes and frequencies) as in the air environment. However, 10- and 25- $\mu \mathrm{m}$ microbeads

Table 1. Release conditions according to objects.

\begin{tabular}{cccc}
\hline Medium & Target Object & Frequency & Amplitude \\
\hline Air & $55 \mu \mathrm{m}$ microbeads & $50,100 \mathrm{~Hz}$ & $4 \sim 9 \mu \mathrm{m}$ \\
& $55 \mu \mathrm{m}$ microbeads & $50,100 \mathrm{~Hz}$ & $4 \sim 9 \mu \mathrm{m}$ \\
Water & $25 \mu \mathrm{m}$ microbeads & $50,100 \mathrm{~Hz}$ & $4.8 \sim 9 \mu \mathrm{m}$ \\
& $10 \mu \mathrm{m}$ microbeads & $100 \mathrm{~Hz}$ & $4.8 \sim 9 \mu \mathrm{m}$ \\
PBS & NIH3T3 cells & $100 \mathrm{~Hz}$ & $4.8 \sim 9 \mu \mathrm{m}$ \\
\hline
\end{tabular}


and 16- $\mu \mathrm{m}$ NIH3T3 cells were not released from the end-effector when the amplitude was lower than $4.8 \mu \mathrm{m}$ at $100 \mathrm{~Hz}$, and even $10-\mu \mathrm{m}$ microbeads and $16-\mu \mathrm{m}$ cells could not be detached when the frequency was $50 \mathrm{~Hz}$ and the amplitudes were from $4.8 \mu \mathrm{m}$ to $9 \mu \mathrm{m}$. From the results in water, it can be seen that, when the size of the microbeads is smaller, the required external forces calculated by the frequency and the amplitude of the end-effector in Equations ((2) and (3)) for release are larger.

\subsection{Release of Microbeads in Air}

Experiments were conducted in different environments such as air, water, and PBS. First of all, the results of release in air are analyzed. In the environment of air, objects can sometimes move rapidly; as a result, objects can become located outside of the visible area. If microbeads $(55-\mu \mathrm{m})$ after release moved inside of the visible area $(512 \mu \mathrm{m} \times 512 \mu \mathrm{m})$, the release task was judged as a success; in the opposite case, the task was considered as a failure. Table 2 shows the success rate of release tasks in air according to the motions and the amplitudes of the end-effector. From these results, 1D motion in the X-and Y-directions can be seen to fail as the amplitude of the end-effector is increased. Moreover, the relationship between the success rate and the frequency can also be established. Lower frequencies of the end-effector show higher success rates for release. On the other hand, 1D motion in the Z-direction and circular motion always succeed in placing microbeads inside the visible area. If the displacement of the end-effector was over $9 \mu \mathrm{m}$, the object was located outside of the visible area at all times.

To analyze the results of release tasks in terms of placement accuracy, the position change after release was measured. Each motion was repeated eight times for statistical analysis. To compare every motion, the mean value and standard deviation of the displacement of the microbeads of the eight repeated motions were computed. Figures 9 (a)-(c) show the results of the experiments. The bars indicate the mean value and the error bars express the standard deviation. 1D motion in the Y-direction was included in the smallest value of each motion because the maximum values of the amplitude of the end-effector in the $\mathrm{Y}$-axis $(1.2$ $\mu \mathrm{m}$ in air and $2.4 \mu \mathrm{m}$ in liquid) were smaller than the smallest values for other motions.

Table 2. Success rate of release tasks in air.

\begin{tabular}{|c|c|c|c|c|c|c|c|}
\hline \multicolumn{2}{|c|}{ Motion } & \multicolumn{6}{|c|}{ Amplitude } \\
\hline & & $1.2 \mu \mathrm{m}$ & $2.4 \mu \mathrm{m}$ & $4 \mu \mathrm{m}$ & $4.8 \mu \mathrm{m}$ & $6 \mu \mathrm{m}$ & $9 \mu \mathrm{m}$ \\
\hline \multirow[t]{2}{*}{$50 \mathrm{~Hz}$} & $\mathrm{X}$-axis & & & $81 \%$ & $73 \%$ & $72 \%$ & $36 \%$ \\
\hline & Y-axis & $100 \%$ & $59 \%$ & & & & \\
\hline \multirow[t]{2}{*}{$100 \mathrm{~Hz}$} & $\mathrm{X}$-axis & & & $73 \%$ & $61 \%$ & $57 \%$ & $35 \%$ \\
\hline & $\mathrm{Y}$-axis & $100 \%$ & $0 \%$ & & & & \\
\hline
\end{tabular}


(a)

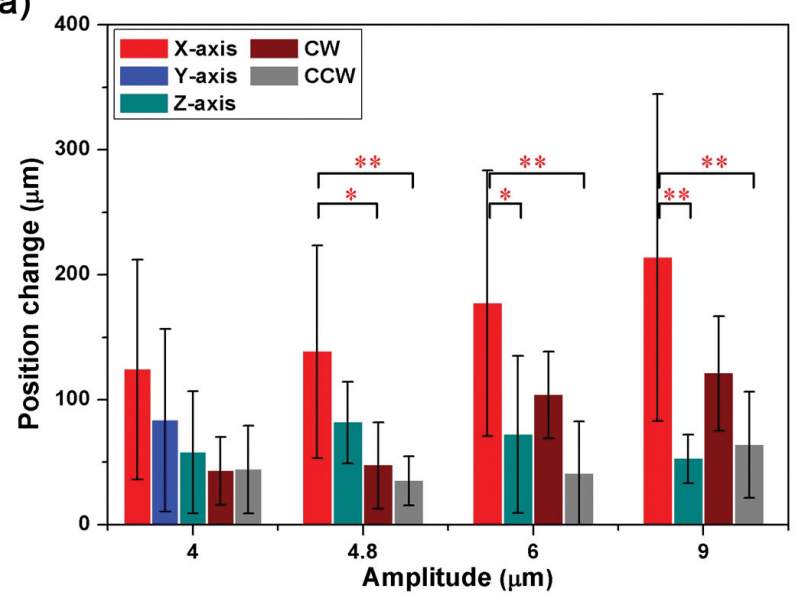

(c)

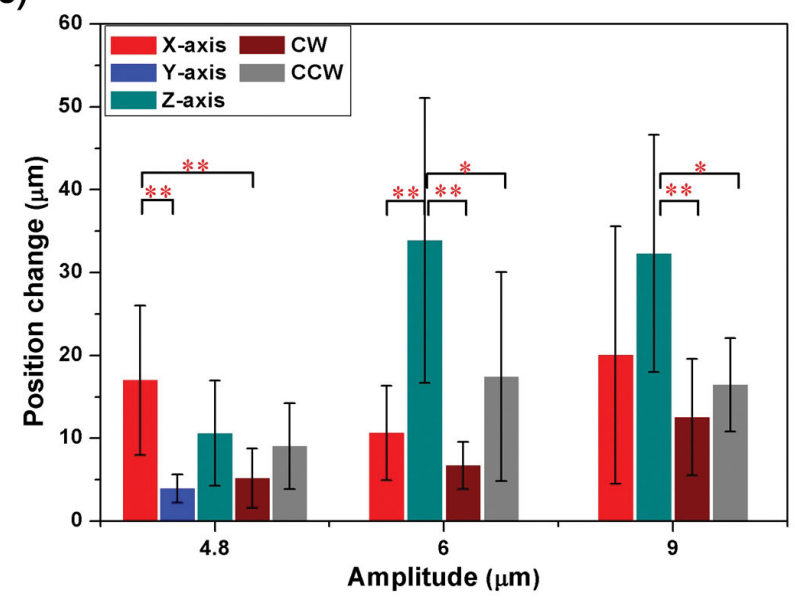

(b)

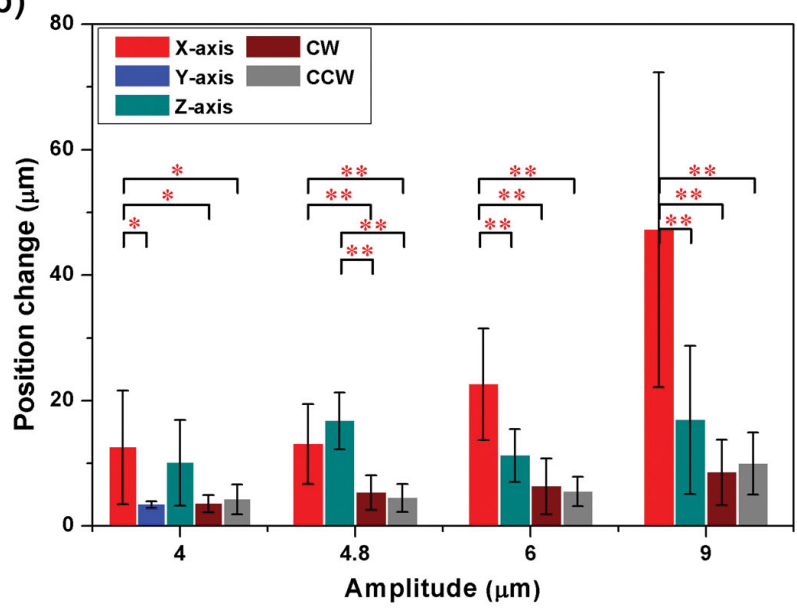

(d)

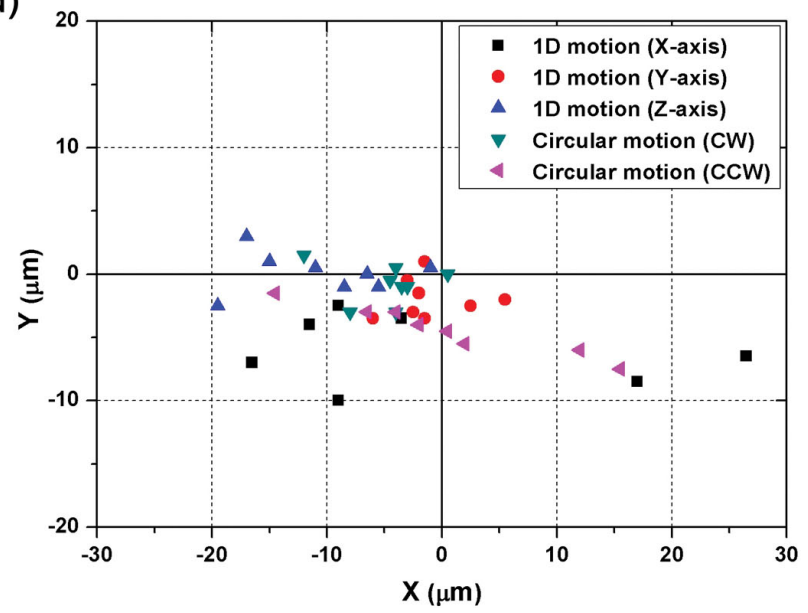

Figure 9. Experiment results (a) position change of $55-\mu \mathrm{m}$ microbeads for motions in air $\left({ }^{*} \mathrm{p}<0.05,{ }^{* *} \mathrm{p}<0.01\right.$ ) (b) position change of $55-\mu \mathrm{m}$ microbeads for motions in water $\left({ }^{*} \mathrm{p}<0.05\right.$, $\left.{ }^{* *} \mathrm{p}<0.01\right)$ (c) position change of NIH3T3 cells for motions in PBS $\left({ }^{*} \mathrm{p}<0.05,{ }^{* *} \mathrm{p}<0.01\right)(\mathrm{d})$ placement position or the applied motions (NIH3T3 cells in PBS)

The position change values of the microbeads for motions in air when the frequency was $100 \mathrm{~Hz}$ are shown in Figure 9(a). Through this graph, 1D motion in the Z-direction and circular motion show more precise placement than $1 \mathrm{D}$ motion in the $\mathrm{X}$ - and $\mathrm{Y}$-directions at the same amplitudes of the end-effector. 1D motion in the Y-direction $(2.4 \mu \mathrm{m})$ is not included since these cases failed to release within the visible area. In this graph, the microbeads detached by using counterclockwise circular motion showed the most precise positioning among the five motions. To verify that counterclockwise circular motions can release objects in the nearest position, we compare five motions. There were no significant differences between 1D motion in the Z-direction and counterclockwise circular motions. Generally, the position accuracy deteriorated with the increase of the amplitude of the end-effector. However, in case of the 1D motion (Z-axis), position accuracy improved as the amplitude increased, which was different from the case for circular motions, for which larger amplitudes showed larger position changes, meaning that position control was more difficult for the 1D motions (Z-axis). Thus, we conclude that counterclockwise circular motion is 
suitable for precise placement in air. The reason for more precise positioning with the counterclockwise (CCW) motion is that the direction of circular motion has an effect on the position of the object. The object moved in the clockwise (CW) direction is pulled upwards and released from the end-effector whereas the counterclockwise circular motion pushes the object downwards. Finally, the object moved in the CCW direction will be located at a closer position to the end-effector than the CW direction in air.

\subsection{Release of Microbeads in Water}

The results of the release task in water are investigated. The same size of microbeads $(55-\mu \mathrm{m})$ was utilized for analyzing the relationship between the environments of air and water. In this case, the microbeads after release were always placed in the visible area; consequently, the success rate was $100 \%$. Figure 9 (b) displays the value of the position change of the microbeads according to the motions at $100 \mathrm{~Hz}$. 1D motion in 1the Y-direction and circular motion showed more precise placement than $1 \mathrm{D}$ motion in the $\mathrm{X}$ - and Z-directions at the same end-effector amplitude. For example, 1D motion in the $\mathrm{X}$ - and Z-directions were significantly different than other motions, whereas three motions (1D motion in the Y-direction and two circular motions) were not significantly different from each other. Although the amplitude of $1 \mathrm{D}$ motion in the Y-direction was too small to compare with other motions, this motion is suitable for successful release. This is because 1D motion in the Y-direction was able to release the object in spite of the small amplitude while the other motions could not detach the same size of object using the same amplitude $(2.4 \mu \mathrm{m})$. Thus, we conclude that the two types of circular motions and the Y-motion are proper motions for precise placement. In addition, from this figure, the relationship between end-effector amplitude and position change can be analyzed. As the amplitude of the end-effector is decreased, microbeads can be located at closer positions to the initial position.

\subsection{Release of NIH3T3 Cells in PBS}

NIH3T3 cells in PBS were utilized to verify the precise release of the proposed motions. Figure 10 demonstrates precise positioning of the released NIH3T3 cells using the clockwise circular motion. As in the case of the water, objects were always located in the visible area. Figure 9 (c) indicates the position change according to motion after release. The results were similar to those in water. Specifically, 1D motion (Y-axis) and the two circular motions were not significantly different. Thus, circular motion and Y-motion were better than the other three motions in terms of position accuracy. Figure 9(d) shows the placement position of the cell after release according to motions when the amplitude of end-effector was $4.8 \mu \mathrm{m}$. From this graph, the analyses of the motions are available by analyzing the range of the placement positions and the relationship between the placement positions of the microbeads and motions. $1 \mathrm{D}$ motions in the $\mathrm{X}$ - and Z-directions as well as counterclockwise circular motion had a wide 
range of placement positions. In contrast, 1D motion in the Y-direction and clockwise circular motion had a small range of placement positions. From the experimental data, we could assume that clockwise circular motion and 1D motion in the Y-direction were better than other motions in terms of object positioning. The reason that the $\mathrm{CW}$ direction would place the objects on the smaller region than the CCW direction is that small object size could have an effect on the local fluid stream generated by the end-effector's motion. The circular motion creates a rotational flow around the end-effector whose direction is the same as the direction of the motion of the end-effector [24] [25]. They observed a relationship between the distance from the center of the rotation and the angular velocity of the microbeads around the end-effector. For example, CW motion of the end-effector creates a clockwise rotational flow, and the flow could push the object to the left side of the end-effector Figure 11(a). On the other hand, CCW circular motion produces a counterclockwise rotational flow, and the stream could pull the object towards the end-effector Figure 11(c). However, the final position of the object depends on the time when the object is released from the end-effector since the performance time of high-speed motion is $100 \mathrm{~ms}$. We could verify the phenomenon through Figure 9(d). In the graph,
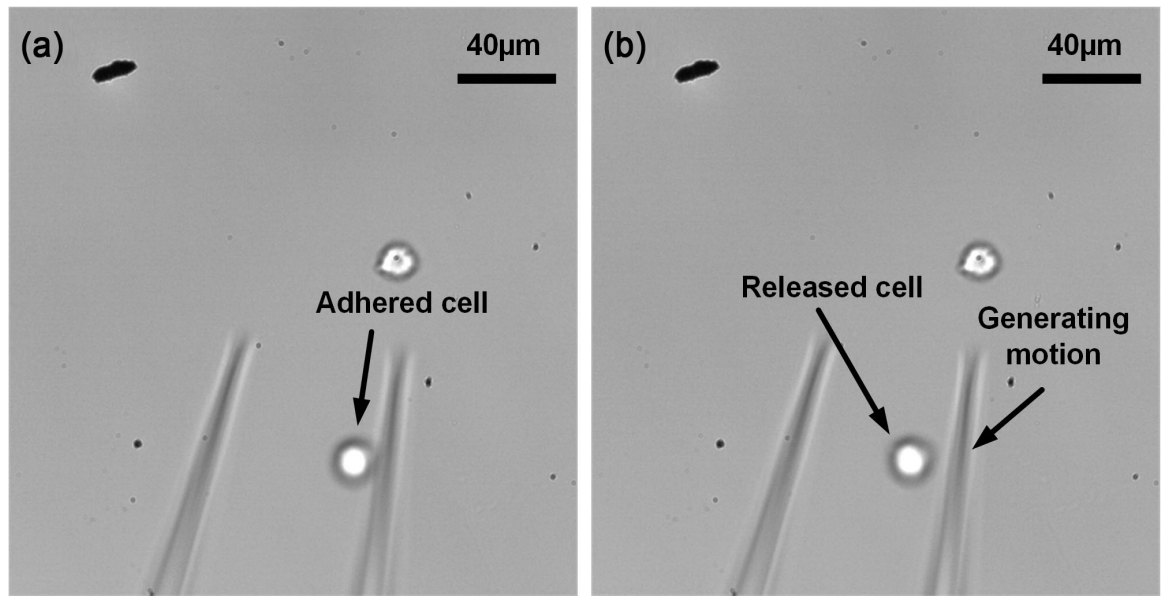

Figure 10. Release of an NIH3T3 cell (a) before release (b) after release.

(a)

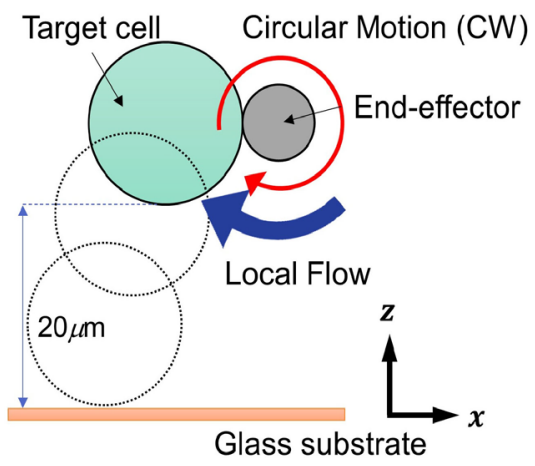

(b)

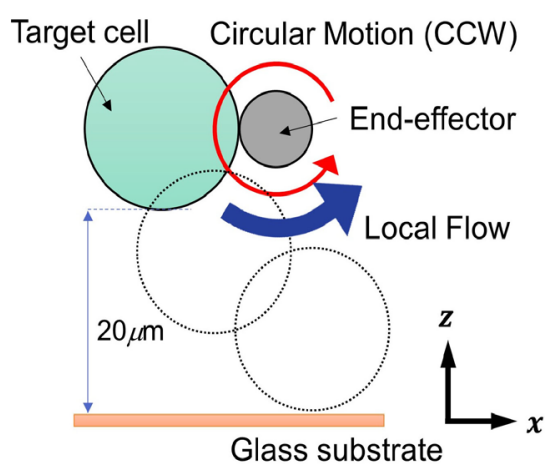

Figure 11. Comparison of two circular motions in PBS (a) clockwise motion (b) counterclockwise motion. 
the objects released by the CW motion were placed on the left side of the desired position, while it the objects detached by the CCW motion were not located on the fixed position. In addition, in the case of clockwise circular motion, small amplitude of the end-effector can guarantee more precise positioning, which is different from the case for other motions.

\subsection{Comparison of Experimental Results in Air and Liquid Environments}

Based on the previous experimental data in different environments (air, water, and PBS), the proposed motions that can improve placement precision after release are verified. To analyze the results of the experiments, at first, different frequencies and amplitudes of the end-effector were examined to ascertain whether release was achieved or not. Then, five motions were applied in order to compare the position accuracy after release. As a result, several outcomes can be obtained.

First, micro-objects in air are located within a larger space than in liquid. To be specific, the objects in air are positioned over $50 \mu \mathrm{m}$ from the initial position and are not even located in visible areas, whereas the values of the position change of objects in liquid are less than $50 \mu \mathrm{m}$. This outcome agrees with the former assumption that the hydrodynamic force in water is stronger than in air owing to the higher dynamic viscosity in the former medium, which hinders the object from moving quickly a short time after separation; finally, the object in water will be located at a closer position than in air.

Second, in every environment, we can find the relationship between the amplitude and the position accuracy: the smaller the amplitude, the better the placement accuracy achieved. This result verified that the external force decided by the amplitude and frequency of the end-effector in Equations (1)-(3) increases with the increase of amplitude. Consequently, the minimum amplitude that is able to detach objects from the end-effector can release micro-objects with high position accuracy.

Finally, for different environments, active release motions with high position accuracy are different. In the case of air, counterclockwise circular motions can release microbeads at the nearest position from the initial position. In the case of water, 1D motion in the Y-direction and circular motions can detach microbeads with small position changes. Lastly, 1D motion in the Y-direction and clockwise circular motions can separate NIH3T3 cells precisely. In the second section, we proposed these motions by analyzing the movement of objects after release. From these experimental results, we verified the assumption that the proposed motions can release and place objects precisely.

\section{Conclusion}

To release micro-objects with high position accuracy, a release method using dynamic motions was proposed. This release method was applied for different 
sizes of objects and different environments (air, water, and PBS). To release micro-objects, the status of a micro-object after release was analyzed in two different environments (air and liquid media). From the analyses, circular motion for the air environment, and circular motion and 1D motion in the Y-direction for the liquid environment are proposed for improving placement accuracy. To verify the efficiency of the proposed motions, five motions, including 1D motion in the X-, Y-, and Z-directions and circular motion (CW, CCW) were applied by comparing the position change after the release of 10-, 25-, and 55- $\mu \mathrm{m}$ microbeads and NIH3T3 cells; we then applied statistical methods. As the result, we verified the fact that the proposed motions can release objects at suitable positions with high position accuracy. In the future, we will extend this approach to the automatic system that can release objects on the precise position.

\section{Acknowledgements}

This work was partially supported by the Grant-in-Aid for Scientific Research (A) (JP16H02321), Grant-in-Aid for Young Scientists (A) (JP16H06076) from the Ministry of Education, Culture, Sports, Science and Technology of Japan.

\section{References}

[1] Fantoni, G. and Porta, M. (2008) A Critical Review of Releasing Strategies in Microparts Handling. International Precision Assembly Seminar, Chamonix, 10-13 February 2008, 223-234.

[2] Saito, S., Miyazaki, H.T., Sato, T. and Takahashi, K. (2002) Kinematics of Mechanical and Adhesional Micromanipulation under a Scanning Electron Microscope. Journal of Applied Physics, 92, 5140-5149.

[3] Fuchiwaki, O., Ito, A., Misaki, D. and Aoyama, H. (2008) Multi-Axial Micromanipulation Organized by Versatile Micro Robots and Micro Tweezers. IEEE International Conference on Robotics and Automation, Pasadena, 19-23 May 2008, 893-898. https://doi.org/10.1109/ROBOT.2008.4543318

[4] Hériban, D. and Gauthier, M. (2008) Robotic Micro-Assembly of Microparts using a Piezogripper. IEEE/RSJ International Conference on Intelligent Robots and Systems, Nice, 22-26 September 2008, 4042-4047. https://doi.org/10.1109/IROS.2008.4650932

[5] Horade, M., Kojima, M., Kamiyama, K., Kurata, T., Mae, Y. and Arai, T. (2015) Development of an Optimum End-Effector with a Nano-Scale Uneven Surface for Non-Adhesion Cell Manipulation using a Micro-Manipulator. Journal of Micromechanics and Microengineering, 25, Article ID: 115002. https://doi.org/10.1088/0960-1317/25/11/115002

[6] Rong, W., Fan, Z., Wang, L., Xie, H. and Sun, L. (2014) A Vacuum Microgripping Tool with Integrated Vibration Releasing Capability. Review of Scientific Instruments, 85, Article ID: 085002. https://doi.org/10.1063/1.4891695

[7] Takahashi, K., Kajihara, H., Urago, M., Saito, S., Mochimaru, Y. and Onzawa, T. (2001) Voltage Required to Detach an Adhered Particle by Coulomb Interaction for Micromanipulation. Journal of Applied Physics, 90, 432-437.

https://doi.org/10.1063/1.1379353

[8] Avci, E., Hattori, T., Kamiyama, K., Kojima, M., Horade, M., Mae, Y. and Arai, T. 
(2015) Piezo-Actuated Parallel Mechanism for Biological Cell Release at High Speed. Biomedical Microdevices, 17, 98. https://doi.org/10.1007/s10544-015-0001-7

[9] Chen, T., Pan, M., Wang, Y., Liu, J., Chen, L. and Sun, L. (2012) Manipulation of Microobjects Based on Dynamic Adhesion Control. International Journal of Advanced Robotic Systems, 3. https://doi.org/10.5772/51507

[10] Demaghsi, H., Mirzajani, H. and Ghavifekr, H.B. (2013) Design and Simulation of a Novel Metallic Microgripper using Vibration to Release Nano Objects Actively. Microsystem Technologies, 20, 65-72. https://doi.org/10.1007/s00542-013-1888-7

[11] Haliyo, D.S., Régnier, S. and Bidaud, P. (2003) Manipulation of Micro-Objects using Adhesion Forces and Dynamical Effects. Experimental Robotics VIII. Springer, Berlin, 382-391.

[12] Landolsi, F. and Ghorbel, F.H. (2015) Modeling of Multisample Nanoplacing. Journal of Dynamic Systems Measurement and Control, 137, Article ID: 024502.

[13] Osawa, S. and Ozaki, K. (2011) Automatic Pickup and Release of Particle by Depth Estimation Method with Micromanipulators for Particle Sorting System. IEEE/SICE International Symposium on System Integration, Kyoto, 20-22 December 2011, 857-862. https://doi.org/10.1109/SII.2011.6147561

[14] Fang, Y. and Tan, X. (2006) A Dynamic JKR Model with Application to Vibrational Release in Micromanipulation. IEEE/RSJ International Conference on Intelligent Robots and Systems, Beijing, 9-15 October 2006, 1341-1346. https://doi.org/10.1109/IROS.2006.281920

[15] Chen, B.K., Zhang, Y. and Sun, Y. (2009) Active Release of Microobjects using a MEMS Microgripper to Overcome Adhesion Forces. Journal of Microelectromechanical Systems, 18, 652-659. https://doi.org/10.1109/JMEMS.2009.2020393

[16] Watanabe, T., Iwasaki, M., Matsumura, H. and Jiang, Z. (2009) Study on Adhesion Force Reduction and State Estimation by Piezo-Transducer. IEEE International Conference on Robotics and Automation, Kobe, 12-17 May 2009, 2211-2216. https://doi.org/10.1109/ROBOT.2009.5152254

[17] Kim, E., Kojima, M., Kamiyama, K., Horade, M., Mae, Y. and Arai, T. (2015) Releasing and Accurate Placing of Adhered Micro-Objects using High Speed Motion of End Effector. IEEE/RSJ International Conference on Intelligent Robots and Systems, Hamburg, 28 September-3 October 2015, 2006-2011. https://doi.org/10.1109/IROS.2015.7353642

[18] Arai, F., Andou, D. and Fukuda, T. (1996) Adhesion Forces Reduction for Micro Manipulation Based on Micro Physics. The 9 th Annual International Workshop on Micro Electro Mechanical Systems, San Diego, 11-15 February 1996, 354-359. https://doi.org/10.1109/MEMSYS.1996.494007

[19] Israelachvili, J.N. (2011) Intermolecular and Surface Forces. Academic Press, Cambridge.

[20] Tomas, J. (2006) Mechanics of Particle Adhesion. Particles on Surfaces, 8, 183-229.

[21] Rollot, Y., Régnier, S. and Guinot, J.-C. (1999) Simulation of Micro-Manipulations: Adhesion Forces and Specific Dynamic Models. International Journal of Adhesion and Adhesives, 19, 35-48. https://doi.org/10.1016/S0143-7496(98)90055-0

[22] Gauthier, M., Régnier, S., Rougeot, P. and Chaillet, N. (2006) Analysis of Forces for Micromanipulations in Dry and Liquid Media. Journal of Micromechatronics, 3, 389-413. https://doi.org/10.1163/156856306777924699

[23] Ejima, T., Ohara, K., Kojima, M., Horade, M., Tanikawa, T., Mae, Y. and Arai, T. (2013) Development of Microhand Utilizing Singularity of Parallel Mechanism. 
IEEE/RSJ International Conference on Intelligent Robots and Systems, Tokyo, 3-7 November 2013, 1525-1530. https://doi.org/10.1109/IROS.2013.6696551

[24] Hayakawa, T., Sakuma, S., Fukuhara, T., Yokoyama, Y. and Arai, F. (2014) A Single Cell Extraction Chip using Vibration-Induced Whirling Flow and a Thermo-Responsive Gel Pattern. Micromachines, 5, 681-696. https://doi.org/10.3390/mi5030681

[25] Kim, E., Kojima, M., Xiaoming, L., Hattori, T., Kamiyama, K., Mae, Y. and Arai, T. (2017) Analysis of Rotational Flow Generated by Circular Motion of an End Effector for 3D Micromanipulation. ROBOMECH Journal, 4, 5.

https://doi.org/10.1186/s40648-017-0074-6 